\title{
Diffuse Global Granulomatous Glomerulonephritis in a Pig
}

\author{
Akihiko SUGIYAMA ${ }^{1)}$, Kiyokazu OZAKI ${ }^{2)}$ and Isao NARAMA²)*

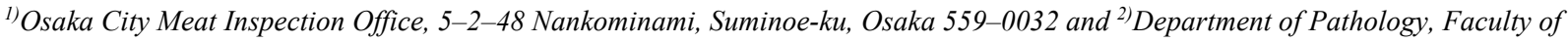 \\ Pharmaceutical Sciences, Setsunan University, 45-1 Nagaotoge-cho, Hirakata, Osaka 573-0101, Japan
}

(Received 9 February 2004/Accepted 27 August 2004)

ABSTRACT. Diffuse global granulomatous glomerulonephritis with unique morphological characters was detected in a pig. The structure of the basement membrane of glomerular tufts was destroyed in almost all glomeruli. Various inflammatory cells consisted mainly of macrophages infiltrated severely into the glomerular tuft and the Bowman's space of and extended to the periglomerular interstitium. Periarteritis with fibrinoid necrosis was occasionally seen in the arterioles and small arteries running through the renal parenchyma and pelvis. In the present case, the results of either the immunohistochemical reactions to the antigens against PRRSV or PCV-2 or ZielNeelsen staining for acid-fast bacilli were negative and no pathogenic bacteria were cultured.

KEY WORDS: global granulomatous glomerulonephritis, periarteritis, swine.

J. Vet. Med. Sci. 67(1): 87-90, 2005

Various morphologic types of glomerulonephritis have been described in pigs $[1,4,10,11]$. We encountered a case of diffuse global granulomatous glomerulonephritis with unique morphological characters in a slaughtered pig and described its detailed morphological features based on the results of a number of examinations for bacteriological and viral infections.

The animal was a 6-month-old, neutered male pig of mixbreed weighting about $100 \mathrm{~kg}$. The pig showed no abnormal clinical signs nor any growth retardation and was diagnosed as apparently healthy from its outer appearance. It was subsequently slaughtered at the Osaka City abattoir.

Macroscopically, both kidneys were markedly enlarged $(20.5 \times 7.0 \times 2.0 \mathrm{~cm}, 17.0 \times 8.0 \times 3.5 \mathrm{~cm})$ with pale cortices and peppered with haemorrhagic foci $2-4 \mathrm{~mm}$ in diameter (Fig. 1). No macroscopic changes were detected in the other organs.

Following the macroscopic examination, tissue samples from kidneys and renal lymph nodes were fixed with $10 \%$ neutral phosphate-buffered formalin solution. Paraffinembedded sections of 4-5 $\mu \mathrm{m}$ thick were stained with hematoxylin and eosin (HE), the PAM, PAS and Ziel-Neelsen's methods for acid-fast bacteria.

Immunostaining was performed using a Histofine SABPO kit (Nichirei Corp., Tokyo, Japan). The primary antibodies were a mouse monoclonal antibody of anti-human myeloid/histiocyte antigen (Mac387) (1:40, DAKO Denmark, Glostrup, Denmark), a rabbit polyclonal antibody against PRRSV strain chiba92-1 (1:8,000), and a biotinylated swine polyclonal antibody against PCV-2 strain Yamagata (IFA: 40960) (1:80). Anti-human myeloid/histiocyte antigen (Mac387) was used as a primary antibody for marker of macrophages [8]. Simple Stain AEC solution (Nichirei Corp., Tokyo, Japan) was used for the peroxidase coloring reaction.

\footnotetext{
* Correspondence to: Narama, I., Department of Pathology, Faculty of Pharmaceutical Sciences, Setsunan University, 45-1 Nagaotoge-cho, Hirakata, Osaka 573-0101, Japan.
}

Using DHL Agar "Nissui” (Nissui Corp., Tokyo, Japan), GAM Agar, Modified "Nissui" (Nissui Corp., Tokyo, Japan) and blood agar with nalidixic acid, we attempted to culture bacteria from affected kidney and renal lymph node.

Histopathologically, extensive inflammatory lesions were seen in almost all glomeruli and periglomerular interstitium with destruction of the normal structure to varying degrees (Fig. 2). The structure of the basement membrane of glomerular tufts was destroyed. Various inflammatory cells which consisted of mainly macrophages with frequent formations of multinuclear giant cells, lymphocytes, and small number of neutrophils and eosinophils severely infiltrated the glomerular tuft, the Bowman's space, and the periglomerular interstitium (Figs. 3, 4, 5). Some affected glomeruli were replaced entirely by infiltrated macrophages, showing a granulomatous appearance (Fig. 4). The affected glomerular tufts were destroyed with fibrin precipitation and hemorrhage. The basement membranes of Bowman's capsule had disappeared partly or completely, and their continuity was lost to varying degrees corresponding to the extent of destruction (Fig. 4). These macrophages and multinuclear giant cells were proved positive for human myeloid/histiocyte antigen (Mac387) (Fig. 6). Hyaline droplet degeneration and regenerative changes in the tubular cells were often seen throughout the cortex (Fig. 7). There were proteinaceous casts, neutrophils, and eosinophils in the tubular lumina near the affected glomeruli. These lesions were less severe in the medulla than in the cortex. Periarteritis with fibrinoid necrosis of blood vessel walls was occasionally seen in the arterioles and small arteries running through the renal parenchyma and pelvis (Fig. 8).

The immunohistochemical reactions to PRRSV or PCV2 in the affected kidney tissue were negative. Bacterial cultures from the kidney and renal lymph node failed to yield any recognized porcine pathogens. The acid-fast bacillus, being positive to the Ziel-Neelsen's staining method, was not detected in either the macrophages or multinuclear giant cells that had infiltrated in the lesions of the affected kidney 

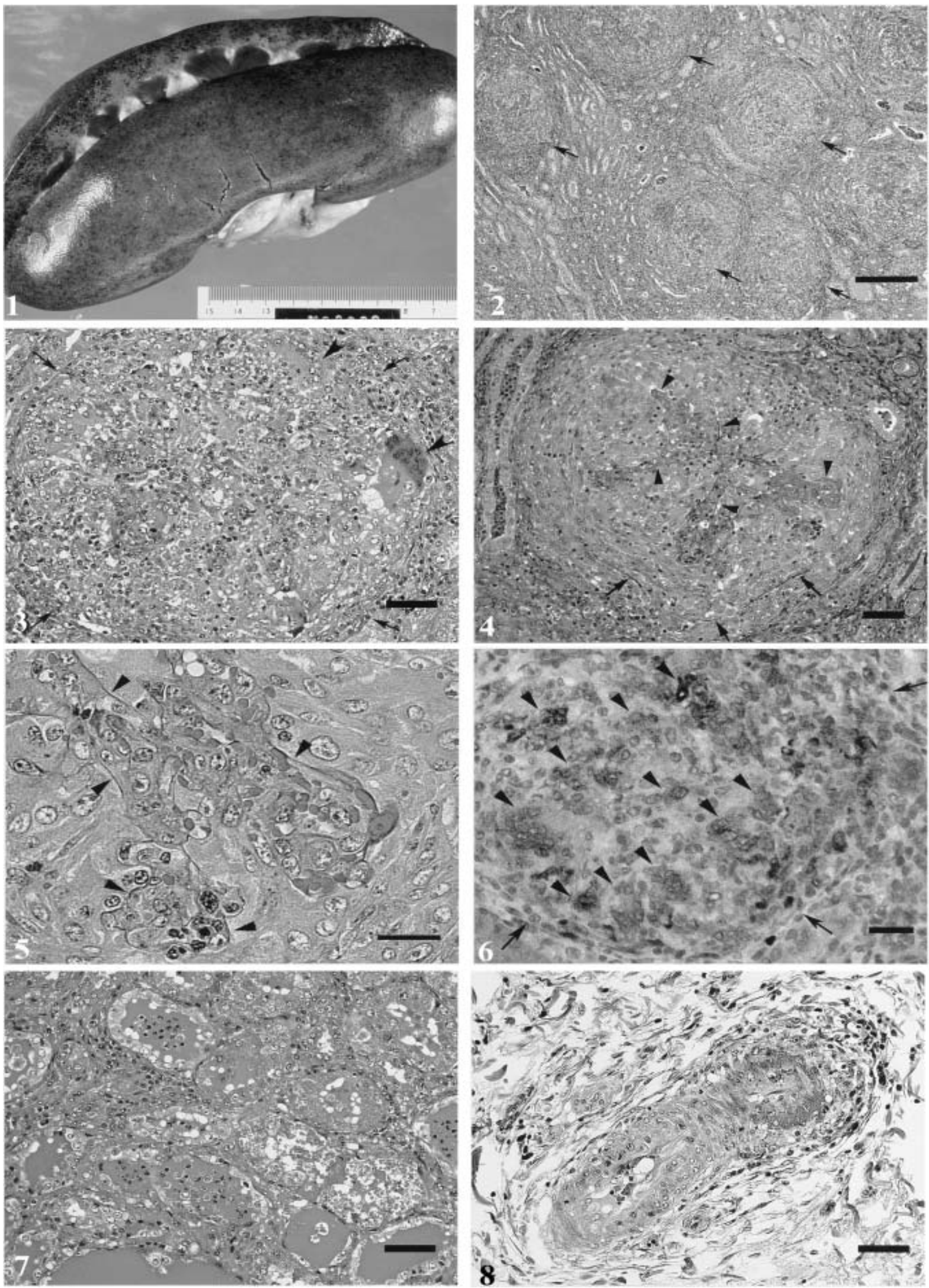
and renal lymph node tissue.

In the present case, the most conspicuous changes were destruction of the structure of the basement membrane of glomerular tufts and severe granulomatous inflammation locating diffusely and globally in the glomeruli, Bowman's space, and periglomerular interstitium. The main inflammatory changes located in the glomeruli. Based on its morphologic character, this renal lesion was diagnosed as a diffuse global granulomatous glomerulonephritis. To the best of our knowledge, this morphologic type of glomerulonephritis is very rare and has not been reported previously in swine.

Anti-GBM glomerulonephritis is known as a class of "granulomatous glomerulonephritis" [3,5]. Although there is a wide spectrum of abnormalities affecting the glomeruli, the most characteristic finding is the presence of circumferential cellular crescents associated with varying degrees of destruction of the capillary tufts and basement membrane of the capillary tuft [3]. The capillary tufts are disrupted, compressed, and distorted as a consequence of further damage of basement membrane and capsular space becomes to be filled with cells such as monocytes and lymphocytes in the chronic course of the disease [3]. The histological characteristics of glomerular lesions in the present case were very similar to those in chronic stage of anti-GBM glomerulinephritis. From the standpoint of characteristic morphological features of glomerular lesions such as destruction of the basement membrane of capillary tufts and granulomatous inflammation seen in the present case, it is likely that this lesion involved a mechanism similar to that of anti-GBM glomerulonephritis. In domestic animals, anti-GBM disease is apparently rare, and reported only in a horse in addition to several suspected but not confirmed cases in dogs [6].

Periarteritis with fibrinoid necrosis of blood vessel walls was occasionally seen in the arterioles and small arteries running through the renal parenchyma and pelvis in the present case. The vascular lesions were mild to medium grade and in acute stage, lacking the proliferative changes of mesenchymal cells. The frequency and stage of the vascular lesions were markedly different from those of glomerular lesions. From these findings, the correlation between vascular lesions and glomerular lesions is unclear.

Porcine dermatitis and nephropathy syndrome (PDNS) have been identified as a swine disease showing glomerulonephritis involving vasculitis in the renal tissue $[9,11]$. However, the morphologic features of glomerular lesions of PDNS are quite different from those found in the present case, since glomerulonephritis of PDNS is characterized by severe necrotizing changes and fibrinous exudates $[7,12]$. In the present case, the immunohistochemical reactions to the antigens against PRRSV or PCV-2 which are involved in PDNS [2, 9], were negative. Consequently, it is unlikely that the present case is associated with PDNS.

Although the actual cause and pathogenic mechanism remain unclear, we considered it noteworthy to describe such a rare lesion in the slaughtered pig.

ACKNOWLEDGMENTS. The authors wish to thank Dr. Makoto Haritani (National Institute of Animal Health) and Dr. Kenji Kawashima (Shichinohe Research Unit, National Institute of Animal Health) for the immunohistochemical staining.

\section{REFERENCES}

1. Bourgault, A. and Drolet, R. 1995. J. Vet. Diagn. Invest 7: 122-126.

2. Choi, C. and Choe, C. 2001. Vet. Pathol. 38: 436-441.

3. Cohen, A. H. and Glassock, R. J. 1989. pp. 494-521. In: Renal Pathology with Clinical and Functional Correlations. Vol. 1 (Tisher, C. C. and Brenner, B. M. eds.), J. B. Lippincott Company, Philadelphia.

4. Jansen, J. H. 1993. A. P. M. I. S. 101: 281-289.

5. Jennette, J. C. 1998. pp. 625-656. In: Heptinstall's Pathology of the Kidney, 5th ed., vol.1 (Jennette, J. C., Olson, J. L., Schwartz, M. M. and Silva, F. G. eds.), Lippincott-Raven, Philadelphia.

6. Maxie, M. G. and Prescott, J. F. 1993. pp. 478-480. In: Pathology of Domestic Animals, vol.2, 4th ed. (Jubb, K. V. F., Kennedy, P. C. and Parmer, N. eds.), Academic Press, New York.

Fig. 1. Macroscopically, the kidney was enlarged with pale disseminated haemorrhagic foci ranging $2-4 \mathrm{~mm}$ in diameter throughout whole cortices.

Fig. 2. Extensive inflammatory lesions were seen in almost all glomeruli (arrows) and periglomerular interstitiums with destruction of the normal structure to varying degrees throughout the renal cortex. HE stain. Bar $=300 \mu \mathrm{m}$.

Fig. 3. Various inflammatory cells which consisted of mainly macrophages with formations of multinuclear giant cells (arrowheads), lymphocytes, and small number of neutrophils and eosinophils severely infiltrated the glomerular tuft, the Bowman's space, and the periglomerular interstitium (arrows: glomerulus). HE stain. Bar $=50 \mu \mathrm{m}$.

Fig. 4. The structure of the basement membrane of glomerular tufts (arrowheads) was destroyed, and inflammatory cells consisted mainly of macrophages that replaced the glomerular tufts and Bowman's space almost completely, and further infiltrated the periglomerular interstitium. The basement membrane of Bowman's capsule (arrows) disappeared partly. PAM stain. Bar $=50 \mu \mathrm{m}$

Fig. 5. The Bowman's space was filled with macrophages, and the structure of the basement membrane of glomerular tufts (arrowheads) was disrupted with hemorrhage. PAM stain. Bar $=20 \mu \mathrm{m}$.

Fig. 6. These macrophages and multinuclear giant cells were positive for human myeloid/histiocyte antigen (Mac387). (arrows: glomerulus, arrowheads: macrophages and multinuclear giant cells) $B a r=50 \mu \mathrm{m}$.

Fig. 7. Hyaline droplet degeneration and regenerative changes in the tubular cells were often seen throughout the cortex. There were proteinaceous casts, and neutrophils, and eosinophils in the tubular lumina. HE stain. Bar=50 $\mu \mathrm{m}$.

Fig. 8. Periarteritis with fibrinoid necrosis of blood vessel walls was seen in small arteries running through the pelvis. HE stain. Bar=50 $\mu \mathrm{m}$. 
7. Molnar, T., Glavits, R., Szeredi, L. and Dan, A. 2002. Acta Veterinaria. Hungarica. 50: 5-16.

8. Perez, J., Garcia, P. M., Mozos, E., Bautista, M. J. and Carrasco, L. 2001. J. Comp. Pathol. 124: 200-206.

9. Segales, J., Piella, J., Marco, E., Mateu-de-Antonio, E. M., Espuna, E. and Domingo, M. 1998. Vet. Rec. 142: 483-486.
10. Shirota, K., Koyama, R. and Nomura, Y. 1986. Jpn. J. Vet. Sci. 48: $15-22$.

11. Smith, W. J., Thomson, J. R. and Done, S. 1993. Vet. Rec. 132: 47.

12. Thomson, J. R., Higgins, R. J., Smith, W. J. and Done, S. H. 2002. J. Vet. Med. 49: 430-437. 